\title{
GATED IR IMAGES OF SHOCKED SURFACES
}

\author{
Stephen S. Lutz ${ }^{1}$, W. Dale Turley ${ }^{1}$, Paul M. Rightley ${ }^{2}$, and Lori E. Primas ${ }^{2}$ \\ ${ }^{1}$ Bechtel Nevada (BN), Special Technologies Laboratory, 5520 Ekwill St., Suite B, Goleta, CA 93117 \\ ${ }^{2}$ Los Alamos National Laboratory (LANL), Los Alamos, NM 87545
}

\begin{abstract}
Gated infrared (IR) images have been taken of a series of shocked surface geometries in tin. Metal coupons machined with steps and flats were mounted directly to the high explosive. The explosive was point-initiated and 500-ns to 1-microsecond-wide gated images of the target were taken immediately following shock breakout using a Santa Barbara Focalplane InSb camera (SBF-134). Spatial distributions of surface radiance were extracted from the images of the shocked samples and found to be non-single-valued. Several geometries were modeled using CTH, a two-dimensional Eulerian hydrocode.
\end{abstract}

\section{INTRODUCTION}

Experimental methods used to determine the state of a shocked material are extensive and in many cases return data with a reported accuracy of a few percent(1). Although significant progress has occurred in the advancement of diagnostic methods in shock experiments, one opportunity for improvement is extension of a single-spatial-point measurement to a high-resolution multipoint image. A number of single-spatial-point studies have been reported describing the measurement of timeresolved temperature change on the surface of a metal subjected to shocks on the order of $100 \mathrm{GPa}$ (2-7). Using the most advanced detectors and radiometric methods, these researchers report the ability to resolve a $100^{\circ} \mathrm{K}$ temperature rise above ambient to a few percent accuracy, with time resolution on the order of $100 \mathrm{~ns}$.

With recent advances in single-pixel detectors have come significant improvements in gated thermal cameras. One such example is the SBF134. This camera is based on an indium antimonide (InSb) detector, bump bonded to a silicon CID array readout structure. With a gate time of $500 \mathrm{~ns}$, we found that $\sim 500$ photons/pixel were required to produce an image with 2:1 signal-to-noise ratio. In practical terms, this camera is able to resolve distinct facial features of a human using a $500 \mathrm{~ns}$ gate width. We used this camera to capture freesurface thermal images of metal samples shocked to pressures on the order of $20 \mathrm{GPa}$. By modifying surface finish and surface structure we were able to affect the apparent residual temperature of the shocked materials. We modeled shock-induced temperature rise and compared the results with the experimental measurements.

\section{EXPERIMENTAL}

Experiments were carried out in a RISI explosive test chamber. Signal from the shocked coupon was reflected off a gold-coated mirror, through a sapphire viewing port and imaged onto the camera focal plane. A sketch of a typical high explosive experiment package is shown in Figure 1. A RP80 detonator single point initiated a two gram charge of Detasheet (Dupont C2-EL-506). The Detasheet charge directly contacted the metal coupon under study. The whole assembly was housed in a black Delrin package.

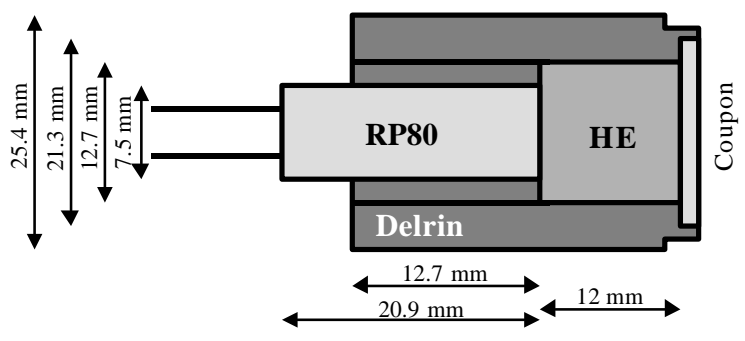

FIGURE 1. Shot package design. 
TABLE 1. Physical Characteristics of Tin Targets and Camera Gate Times; $t_{\mathrm{o}}$ is Shock Breakout Time

\begin{tabular}{|c|c|c|c|c|c|c|}
\hline \multirow[b]{2}{*}{$\#$} & \multirow[b]{2}{*}{ Geometry } & \multirow[b]{2}{*}{ Surface Finish } & \multirow{2}{*}{$\begin{array}{c}\text { Step } \\
\text { Height } \\
(\mathbf{m m})\end{array}$} & \multicolumn{3}{|c|}{$\begin{array}{c}\text { Camera open and close gate times relative } \\
\text { to shock breakout (ns) }\end{array}$} \\
\hline & & & & $\begin{array}{c}\text { Thin Step } \\
\text { (open,close) }\end{array}$ & $\begin{array}{c}\text { Thick Step } \\
\text { (open,close) }\end{array}$ & $\begin{array}{c}\text { Flat } \\
\text { (open,close) }\end{array}$ \\
\hline 1 & Step & Machine finish (\#8) & 1.0 & $\mathrm{t}_{0}+40, \mathrm{t}_{0}+540$ & $\mathrm{t}_{0}-230, \mathrm{t}_{0}+270$ & \\
\hline 2 & Flat & Polished $w / 3 \mu \mathrm{m} \mathrm{Al}_{2} \mathrm{O}_{3}$ & & & & $\mathrm{t}_{0}-230, \mathrm{t}_{0}+270$ \\
\hline
\end{tabular}

Metal samples were prepared with three different surfaces: single step, periodically spaced grooves, and flat polish. Although a variety of geometries were studied, only single step and flat polished tin $(99.9 \%)$ data are presented in this paper. The details of sample preparation and IR camera gate times (open and close), relative to shock breakout, are summarized in Table 1. All metal samples were nominally $2 \mathrm{~mm}$ thick and $21 \mathrm{~mm}$ in diameter.

\section{Camera (SBF-134) Setup and Calibration}

General performance characteristics of the SBF-134 are summarized in Table 2. A detailed temperature calibration was performed using a blackbody source placed in the explosive chamber at the target position. However, for this publication radiance data was not temperature converted. We intend to present temperature calibrated data in a future report.

\section{Pressure Calibration and Timing Measurements}

The pressure generated by the explosive in the metal coupon was calculated from Hugoniot and measured shock velocity data. The shock velocity was obtained by measuring the time required for the shock wave to travel across a $1 \mathrm{~mm}$ step, machined in a test sample. The step was coated with a thin $(<25 \mu \mathrm{m})$ film sensor that produces a prompt ( $<10 \mathrm{~ns}$ rise) burst of light when shocked. This thin film is a mixture of cerium doped lutetium orthosilicate (LSO:Ce) phosphor powder suspended in a silica glass binder. An optical fiber positioned $2 \mathrm{~mm}$ from the sample surface simultaneously views the high and low surfaces of the step. When the shock arrives the sensor emits a burst of light that is detected with a photomultiplier tube and recorded with a transient digitizer. Using this method, a pressure of $19.7 \mathrm{GPa}$ was determined for the tin experiments.

For each gated imaging experiment, a 3-mmdiameter spot of the previously-discussed shock arrival sensor was applied approximately $5 \mathrm{~mm}$ from the target center. Shock-induced light from this sensor was recorded simultaneously with the high explosive and camera triggers. Such cross timing is essential to interpret the acquired IR image. The jitter between the trigger used to initiate the high explosive and the time of arrival of the shock at the target surface was found to be on the order of $200 \mathrm{~ns}$.

\section{RESULTS}

Figures 2 and 3 show a set of images of samples 1 and 2 in Table 1. In each of these figures, a gated IR image of the shocked target surface (a) is displayed next to a white light image of the preshocked sample (b) taken with a digital camera. A "log counts" grey scale map is used to display the 14-bit digitizer range and to highlight the subtle features in the shocked metal surface.

Figure 2 (a) shows the results from the stepped tin. Notable features include high radiance along the step boundary, and an offset in radiance between the thin and thick steps. The bright circular feature is the high-emissivity phosphor LSO:Ce sensor. The semi-circular feature marking the outer edge of the lower half of the coupon results from motion of the thinner step, presumably with hot gasses escaping from around the edge.

TABLE 2. Camera Performance Characteristics

\begin{tabular}{ccccccc}
\hline Detector & Array Size & $\begin{array}{c}\text { Pixel Size } \\
(\boldsymbol{\mu} \mathbf{~ m})\end{array}$ & $\begin{array}{c}\text { Quantum Efficiency } \\
(\mathbf{3 - 5} \boldsymbol{\mu m})\end{array}$ & $\begin{array}{c}\text { Bit } \\
\text { Depth }\end{array}$ & $\begin{array}{c}\text { Photons/Pixel } \\
\text { Required for 2:1 SNR }\end{array}$ & $\begin{array}{c}\text { Minimum Gate } \\
\text { Width (ns) }\end{array}$ \\
\hline $\mathrm{InSb}$ & $256 \times 256$ & 30 & $\sim 85 \%$ & 14 & 500 & 140 \\
\hline
\end{tabular}



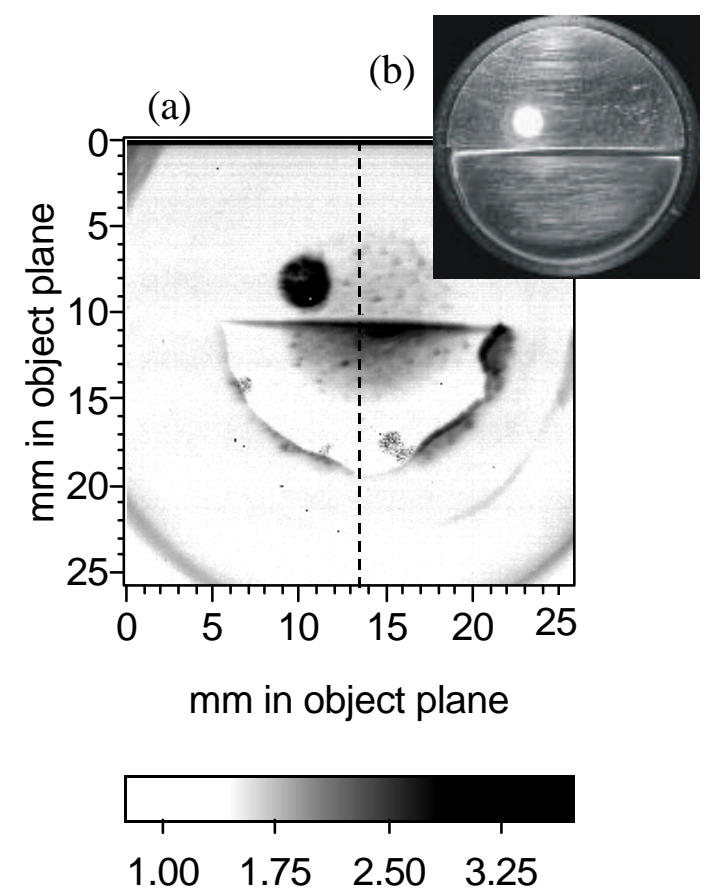

log counts

FIGURE 2. (a) IR image of shocked stepped tin coupon; (b) preshot picture of test coupon.

Figure 3 (a) shows the results from polished tin. Most notable is the variance in the radiance field. Distinct circular features, some hot and some cold, relative to the mean, are clearly evident. The variance is far in excess of the statistical limit imposed by the detection system. Figures 4 and 5 show normalized radiance values taken from locations marked by dashed lines in Figures 2(a) and 3(a).

\section{MODELING}

We have simulated the tin experiments using Sandia National Laboratories' Eulerian mesh CTH code. Our rectangular, 2-dimensional modeling included the Detasheet, Delrin and tin, but did not include the actual detonator geometry (Figure 1). A detonation wave was started at a point corresponding to the center of the output area of the detonator. The equation of state for the explosive products was approximated using JWL parameters for PETN (the principle component of Detasheet). Mie-Grûneisen equation of state data were used for Delrin and tin. Delrin was modeled as an elastic,
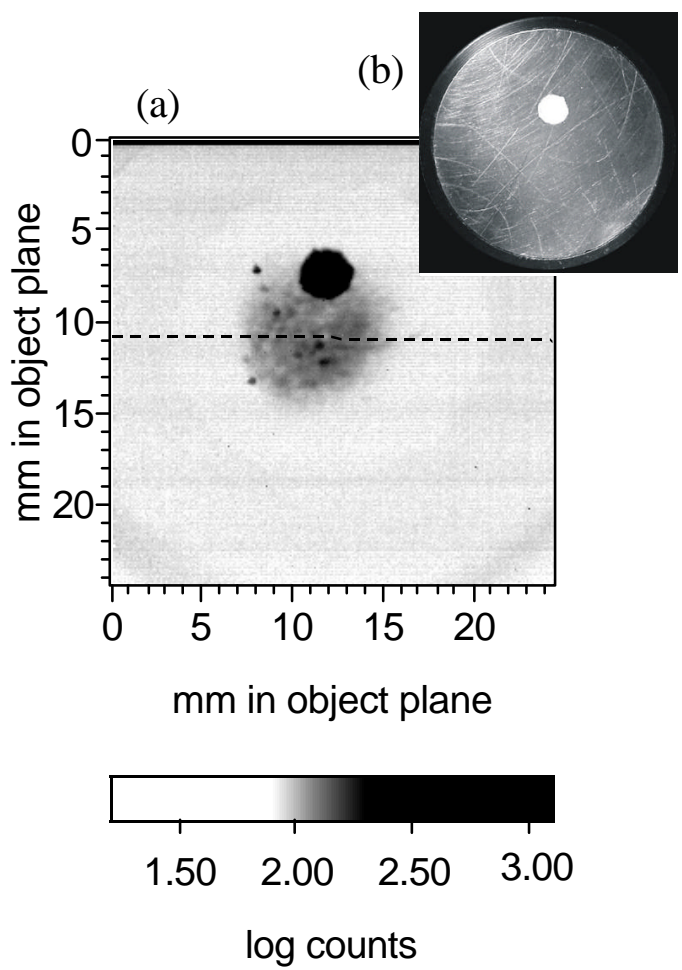

FIGURE 3. (a) IR image of shocked polished tin coupon; (b) preshot picture of test coupon.

perfectly plastic material while a Steinberg-GuinanLund strength model was used for tin.

Lagrangian tracer particles were placed into the simulation at equal intervals along the radius just under the surface of the coupon. The depth of the tracers was approximately one mesh cell below the surface for both samples. A point approximately one third of the way from the center of the coupon (approximating the location of the breakout sensor) was used to determine breakout timing. Breakout was considered to occur when the temperature at this point rose from ambient to $300^{\circ} \mathrm{K}$. Time histories of temperature at each tracer were produced using the experimental timing of the camera gate relative to break-out. To compare the modeled temperature with the measured radiance data it was necessary to integrate the radiance estimated from the modeled temperatures over the duration of the camera gate. Figures 4 and 5 show normalized experimental results over-plotted with the corresponding CTH data. 


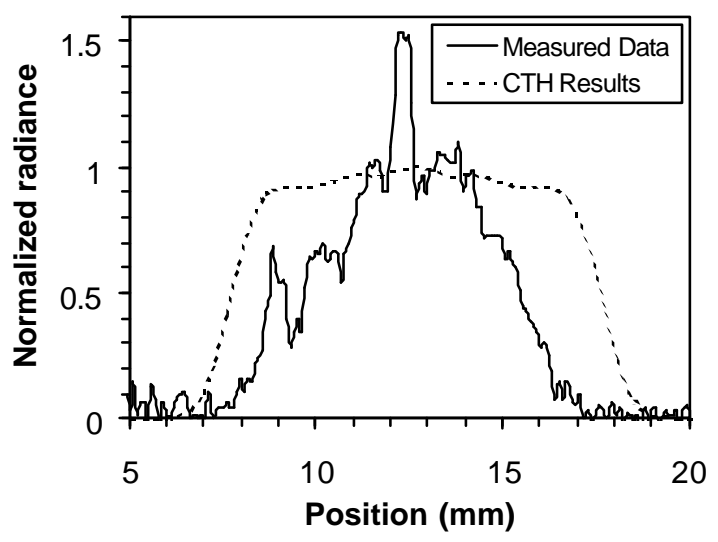

FIGURE 4. Sample 1, polished tin, measured and modeled results.

\section{CONCLUSION}

The large-scale features of the CTH calculations are in general agreement with the radiance data. However, CTH calculations do not account for the magnitude of the variations seen in the radiance data on a sub-millimeter scale. At this scale, differences between the modeled and measured results may be due to grain structure or other features not accounted for in the model. The wealth of data obtained from two-dimensional radiance measurements over a wide range of spatial scales is important to understanding the accuracy and precision limitations of single-point pyrometry measurements. Further, this technique may provide impetus for creation and validation of new constitutive models.

\section{ACKNOWLEDGEMENTS}

We would like to thank Arn Adams of Santa Barbara Focalplane for loan of a SBF-134 camera, Mike Grover and Guy Leach of BN for technical support, Ron Boat of LANL for design of the explosive package, and Dave Holtkamp and Mark Wilke of LANL for review and critique of the overall experiment.

This work was supported by the Department of Energy, Nevada Operations Office, under Contract No. DE-AC08-96NV11718. DOE/NV/11718-542. LA-UR-01-3319. Reference herein to any specific commercial product, process, or service by trade name, trademark, manufacturer, or otherwise, does not necessarily constitute or imply its endorsement, recommendation, or favoring by the United States Government or any agency thereof or its contractors

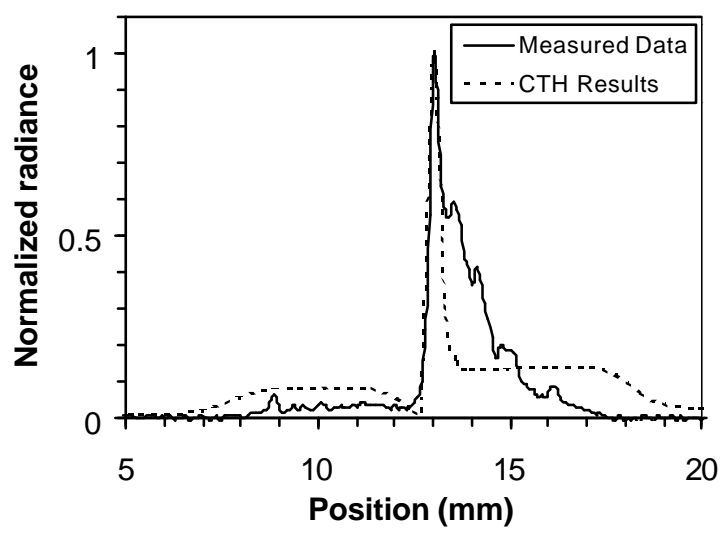

FIGURE 5. Sample 2, stepped tin, measured and modeled results.

or subcontractors. The views and opinions of authors expressed herein do not necessarily state or reflect those of the United States Government or any agency thereof.

\section{REFERENCES}

1. Asay, J.R., and Shahinpoor, M., High-Pressure Shock Compression of Solids, Springer-Verlag, New York, 1993, pp 43-70.

2. Taylor, J.W., "Residual Temperatures of Shocked Copper,” J. Appl. Phys. 43(9), 2727-2731 (1963).

3. Von Holle, W.G., and Trimble, J.J., "Temperature Measurement of Shocked Copper Plates and Shaped Charge Jets by Two-Color IR Radiometry," J.Appl. Phys. 47(6), 2391-2394 (1976).

4. Von Holle, W. G., "Shock Wave Diagnostics by Time-Resolved Infrared Radiometry and Non-Linear Raman Spectroscopy," in Shock Waves in Condensed Matter-1983, edited by J.R. Asay, et. al., Elsevier Science B.V., 1984, pp. 283-291.

5. Perez, M., "Residual Temperature Measurements of Shocked Copper and Iron Plates by Infrared Pyrometry", in Shock Compression of Condensed Matter-1991, edited by S.C. Schmidt, et. al., Elsevier Science B.V., 1992, pp. 737-740.

6. Blanco, E., Remiot, C., Mexmain, J., Herve, P., "Temperature of Shocked Materials Measured with an Infrared Pyrometer through a Window," in Infrared Technology and Applications XXII-1996, edited by B.F. Andresen et. al., SPIE proceedings \#2744, pp. 677-683.

7. Mabire, C., Hereil, P., "Shock Induced Polymorphic Transition and Melting of Tin," in Shock Compression of Condensed Matter-1999, edited by M.D. Furnish, et. al., AIP Conference Proceedings, 2000, pp.93-96. 